\title{
Fabrication of Core-Shell PLGA-Chitosan Microparticles Using Electrospinning: Effects of Polymer Concentration
}

\author{
Nguyen Thi Hiep, ${ }^{1}$ Nguyen Dai Hai, ${ }^{2,3}$ and Vo Van Toi ${ }^{1}$ \\ ${ }^{1}$ Tissue Engineering and Regenerative Medicine Laboratory, Department of Biomedical Engineering, \\ International University, Vietnam National University, Ho Chi Minh City 700000, Vietnam \\ ${ }^{2}$ Institute of Applied Materials Science, Vietnam Academy of Science and Technology, \\ 01 Mac Dinh Chi, District 1, Ho Chi Minh City, Vietnam \\ ${ }^{3}$ Graduate University of Science and Technology, Vietnam Academy of Science and Technology, Hanoi, Vietnam \\ Correspondence should be addressed to Nguyen Thi Hiep; nthiep1981@gmail.com
}

Received 25 April 2017; Accepted 6 July 2017; Published 3 August 2017

Academic Editor: Zhe Qiang

Copyright (C) 2017 Nguyen Thi Hiep et al. This is an open access article distributed under the Creative Commons Attribution License, which permits unrestricted use, distribution, and reproduction in any medium, provided the original work is properly cited.

This investigation aims to fabricate the core-shell microparticles composed of poly(lactic-co-glycolic acid) and chitosan (PLGACS MPs) using electrospinning. The challenge of using electrospinning is that it has many parameters which change product outcome if any single parameter is changed. However, the advantage of this system is that we can fabricate either micro/nanofibers or micro/nanoparticles. To learn about the effect of liquid concentration, the electrospinning parameters (voltage, needle sizes, distance from needle to collector, and ejection speed) were fixed while the concentration of PLGA or chitosan was varied. The results showed that PLGA microparticles can be fabricated successfully when the concentration of PLGA is smaller than $10 \mathrm{wt} \%$. Presence of the chitosan shell was confirmed by zeta potential measurements, FT-IR, optical observation, and fluorescence observation. Thickness of the chitosan shell can be controlled by changing the concentration of chitosan and measured by fluorescamine labeling method. Moreover, SEM observation showed that concentration of chitosan affected the size of PLGA-CS microparticles. The MTT (3-(4,5-dimethylthiazol-2-yl)-2,5-diphenyl tetrazolium bromide) assay test showed that PLGA-CS microparticles possess excellent biocompatibility.

\section{Introduction}

Poly(lactic-co-glycolic acid) microparticles (PLGA MPs) attract considerable therapeutic interest in drug and antigen delivery systems [1-3], mainly because of their sustained release, localized release, and vivo stabilization [3]. However, PLGA is a hydrophobic polymer. Thus, it is difficult to be submerged into water. So, a hydrophilic surface modification is needed to improve the effectiveness of microparticulate delivery systems. To modify the PLGA MPs surface, Pluronics $^{\circledR}$ [4] or chitosan was suggested as a suitable materials to coat conventional PLGA particles [5-8]. Chitosan is normally selected to coat PLGA MPs for drug/genes delivery system because of its hydrophilic property, cationic charge, biodegradability, and mucoadhesive properties [6-10]. There are many ways to coat PLGA surface with chitosan such as solvent double or triple emulsion method [11], static micromixer of $\mathrm{O} / \mathrm{W}$-dispersion of PLGA and chitosan $[5,12]$, droplet microfluidic solvent evaporation and extraction [13], and supercritical fluid technology [14]. Among these methods, the double or triple emulsion method is a conventional approach to coat chitosan onto PLGA microparticles [15]. However, this method has some disadvantages such as (1) organic solvents and shear stress can denature or deactivate the PLGA partly during the preparation process; (2) PLGA particles also generate an acidic internal environment after they gradually hydrolyzed into lactic acid and glycolic acid, which is detrimental to biopolymer stability; and (3) the encapsulation efficiency of PLGA in the particles is essentially low, thus resulting in the consumption of a large quantity of PLGA in the preparation. 
TABLE 1: Parameters of electrospinning.

\begin{tabular}{lcccccc}
\hline & PLGA 85/15 (wt\%) & Chitosan (CS, wt\%) & Needle (gauge) & Distance (cm) & Voltage (kV) & $\begin{array}{c}\text { Ejection speed (ml/h) } \\
\text { PLGA }\end{array}$ \\
\hline PLGA1 & 5 & - & 25 & 15 & 27 & 0.5 \\
CLGA2 & 7 & - & 25 & 15 & - & 27 \\
PLGA3 & 10 & - & 25 & 15 & 27 & 0.5 \\
PLGA-CS1 & 5 & 0.01 & 25 & 15 & 25 & 0.5 \\
PLGA-CS2 & 5 & 0.15 & 25 & 15 & 25 & 0.5 \\
PLGA-CS3 & 5 & 0.3 & & & 0.5 & 0.5 \\
\hline
\end{tabular}

Electrosprayer is a well-controlled method to generate PLGA microparticles $[4,16]$. Surface hydrophilization of hydrophobic PLGA MPs using electrosprayer has been reported by blending with hydrophilic polymer, Pluronic [4]. Moreover, electrosprayer has been known as a high technology to fabricate core-shell electrospun fibers using the core-shell spinneret needle [16-18]. Thus, the purpose of this study is to employ electrospinning machine in fabricating the core-shell poly(lactic-co-glycolic acid)-chitosan microparticles (PLGA-CS MPs) with the spinneret needle's assistance. The advantage of this approach in comparison with conventional methods is the easiness to modify the hydrophilic chitosan onto the hydrophobic PLGA MPs and the thickness of chitosan by changing the concentration of the polymer. In addition, this process, unlike conventional emulsion, prevents polymer waste in washing steps. Solvents are removed during operation time.

The main goal of this investigation is to study about the effect of PLGA and chitosan concentration on fabricating PLGA-CS MPs. Morphology of PLGA-CS MPs was observed by SEM. FT-IR spectrum, zeta potential, and fluorescent observation were employed to evaluate the coating achievements. The biocompatibility of PLGA-CS microparticles was evaluated by cytotoxicity test and cell viability test. This approach is not only fast and safe but also has a high potential for biomedical applications.

\section{Materials and Methods}

2.1. Materials. Chitosan (C3646, $\geq 75 \%$ deacetylated), poly(lactic-co-glycolic) acid (PLGA) ( $85: 15)$, hexamethyldisilazane (HDMS), tetrahydrofuran (THF, minimum 99\%), and dimethylformamide (DMF, 99\%) were purchased from Sigma-Aldrich, USA. Acetic acid $\left(\mathrm{CH}_{3} \mathrm{COOH}, 99.0 \%\right)$ was purchased from Duksan Pure Chemical Co., Korea. Glutaraldehyde was purchased from DaeJung Co., Korea. Dimethylsulfoxide (DMSO, 99.0\%, Samchun Pure Chemical Co., Ltd., Korea), ethanol (Merck, Germany), fetal bovine serum (FBS), PS (penicillin/streptomycin (antibiotics)), Dulbecco's phosphate buffered saline (D-PBS) without calcium or magnesium, 3-(4,5-dimethylthiazol-2-yl)-2,5-diphenyl tetrazolium bromide (MTT), and trypsin-EDTA were purchased from GIBCO (Carlsbad, CA). The L-929 cells line was obtained from ATCC, USA.

\subsection{Methods}

2.2.1. Fabrication of PLGA-CS MPs. Before applying polymer solutions in the electrospinning system, chitosan solutions
TABLE 2: Particles size and zeta potential of PLGA-CS MPs.

\begin{tabular}{lcc}
\hline System & Particles size $(\mu \mathrm{m})$ & Zeta potential $(\mathrm{mV})$ \\
\hline PLGA1 & $0.2-1$ & $-4.8 \pm 0.1$ \\
PLGA2 & $0.2-2$ & $-4.6 \pm 0.2$ \\
PLGA3 & - & $-4.6 \pm 0.2$ \\
PLGA-CS1 & $0.2-3$ & $+14.9 \pm 0.5$ \\
PLGA-CS2 & $2-10$ & $+19.1 \pm 0.4$ \\
PLGA-CS3 & $3-20$ & $+37.0 \pm 0.4$ \\
\hline
\end{tabular}

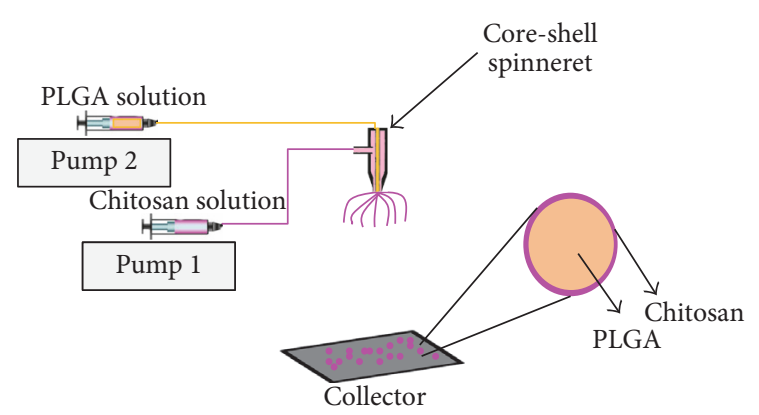

FIGURE 1: Scheme of electrospinning process for fabrication of microparticles.

were prepared by dissolving chitosan in $1.5 \mathrm{wt} \%$ acetic acid where PLGA solutions were prepared in THF:DMF with ratio $1: 1$. Then, the PLGA-CS MPs were formed by ejecting chitosan solution into the shell of the core-shell spinneret needle while PLGA solution was ejected to the core of spinneret as shown in Figure 1. To investigate the effect of the concentration of polymer, other parameters of electrospinning such as the voltage, the distance from needle to collector, and the speed of ejecting solution were fixed (see Table 1).

2.2.2. Characterization of PLGA-CS MPs. The morphology of the core-shell PLGA-CS MPs was examined using scanning electron microscope (SEM, SM-65F, JEOL, Japan). The particle size distribution was determined by counting on SEM images (see Table 2).

Presence of chitosan on PLGA surface was detected by the zeta potential measurement, the FT-IR analysis, the fluorescent observation, the optical observation, and fluorescamine method. The zeta potential measurements of PLGA MPs and PLGA-CS MPs were taken using the zeta potential analyzer. The infrared spectra of PLGA-CS MPs were analyzed by attenuated reflectance Fourier transform spectroscopy (FTIR) (Spectrum GX, PerkinElmer, USA) over a wavelength 
range between 4,000 and $500 \mathrm{~cm}^{-1}$, and the spectra were collected from 64 scans with a resolution of $4 \mathrm{~cm}^{-1}$. Optical images were observed by immersing MPs in water for 24 hours prior to taking photos using a microscope. To obtain fluorescent chitosan, the PLGA-CS MPs were immersed in a $0.1 \mathrm{mg} / \mathrm{ml}$ solution of tetramethylrhodamine (TRITC) in ethanol/water/sodium bicarbonate $(0.1 \mathrm{M})$ and allowed to react for $24 \mathrm{~h}$. Optical images and confocal images of PLGA-CS MPs were observed with an inverted microscope (Leica, Germany) and a confocal microscope (Olympus, FV 1000). To determine the amount of chitosan coated on the surface of PLGA-CS MPs, fluorescamine (Acros Organics; Morris Plains, NJ) was applied [19]. Briefly, the PLGA-CS suspension was obtained by adding $50 \mathrm{mg}$ PLGA-CS MPs to $100 \mu \mathrm{l}$ distilled water. Then, the PLGA-CS suspensions were centrifuged at $16,000 \mathrm{~g}$ in a microcentrifuge (Microcentrifuge, Thermo Scientific). $100 \mu \mathrm{L}$ fluorescamine in DMSO solution $(2.0 \mathrm{~g} / \mathrm{L})$ was added to $20 \mu \mathrm{L}$ of the supernatant in a black 96-well fluorescent detection microplate. After a three-hour reaction in a dark room, fluorescence was measured at an excitation wavelength of $390 \mathrm{~nm}$ and emission wavelength of $515 \mathrm{~nm}$ using a microplate spectrofluorometer reader. A chitosan calibration curve was run simultaneously with each experiment. The amount of chitosan attached to the PLGACS MPs was calculated by dissolving PLGA-CS MPs in acetic acid. The fluorescence intensity was converted to the concentration of chitosan by comparison with a standard curve of pure chitosan. Then, the mass of chitosan coated PLGA-CS MPs was determined as shown in Figure 5.

\subsubsection{In Vitro Experiments}

Sample Sterilization. In order to prepare samples for in vitro experimental processes, the microparticles were placed on an aluminum foil and sterilized with UV irradiation for 15 minutes.

Cell Maintenance. The fibroblast cells (L-929) were subcultured in RPMI-1640 medium and supplemented with 10\% fetal bovine serum (FBS), 1\% penicillin-streptomycin (P/S), and $5 \% \mathrm{FBS}$. Cells were incubated at $37^{\circ} \mathrm{C}$ in a humidified atmosphere of $5 \% \mathrm{CO}_{2}$ (incubator, ASTEC, Japan). Cells were dissociated with trypsin-DETA (GIBCO), centrifuged, and then resuspended in medium. The culture medium was changed every 2 days, supplying enough nutrients to keep cells healthy.

Cytotoxicity Test. To confirm the biocompatibility of microparticles, the cytotoxicity of PLGA, PLGA-CS1, PLGA-CS2, and PLGA-CS3 MPs was evaluated using MTT assay method described in our previous reports [20-24]. Briefly, the fibroblast cells were seeded with a density of $1 \times 10^{4}$ cells $/ \mathrm{ml}$ in a 96-well tissue culture plate and incubated for $24 \mathrm{~h}$ at $37^{\circ} \mathrm{C}$ and $5 \% \mathrm{CO}_{2}$. Diluted extract solutions $(200 \mu \mathrm{l})$ of every well at various concentrations $(100,50,25,12.5$, and $0 \%)$ were then added. The fibroblast cells were treated for 7 days and then $20 \mu \mathrm{l} \mathrm{of} 5 \mathrm{mg} / \mathrm{ml} \mathrm{3-(4,5-dimethylthiazol-2-yl)-2,} \mathrm{5-}$ diphenyltetrazolium bromide (MTT) solution was added to each well. The plate was then incubated for $4 \mathrm{~h}$. After the medium containing MTT solution was removed, DMSO was added to each well to dissolve any insoluble formazan crystals. The optical density (OD) was measured at the wavelength of $595 \mathrm{~nm}$ with an enzyme-linked immunosorbent assay (ELISA) reader (Infinite F50, Tecan Austria GmbH, Austria).

Cell Proliferation Test. In order to evaluate cell proliferation, the fibroblast cells L929 (at a cell density of $10^{4}$ cells $/ 1 \mathrm{ml}$ in RPMI-1640) were seeded on PLGA and PLGA-CS MPs and cultured for various times at $37^{\circ} \mathrm{C}$ in humidified atmosphere with $5 \% \mathrm{CO}_{2}$. Then, cell proliferation was examined using MTT assay $[23,24]$.

\section{Results and Discussion}

PLGA-CS microparticles have been applied for biomacromolecule delivery because of their advantages such as the sustainable and controllable release of PLGA synthesized biopolymer, their cationic charge, biodegradability and mucoadhesive properties of natural chitosan $[6,25]$. So far, there are several methods used for fabricating PLGA-CS MPs; $\mathrm{W} / \mathrm{O}$ emulsion is kind of a famous method [1]. However, W/O method is a consumable method where both biopolymer and drug are easy to be lost in the fabricating process. Hence, we are trying to fabricate PLGA-CS MPs by using electrospinning. Electrospinning is a simple and easy method for fabricating nano/microfibers but it has many parameters to be controlled. Thus, in this investigation, the electrospinning parameters were fixed as mentioned in Table 1 while the concentration of polymers was changed. The purpose of this investigation is to obtain microparticles from electrospinning machine. So the applied voltage of electrospinning machine was changed due to the changing polymer solutions. For fabricating of PLGA MPs, the voltage was $27 \mathrm{kV}$; this high voltage was suitable to obtain the particles; otherwise, the fibers formed. For fabricating the core-shell PLGA-CS MPs, the suitable voltage was $25 \mathrm{kV}$.

To investigate the effect of PLGA concentration on fabricating PLGA MPs, the following electrospinning parameters were fixed as follows: voltage: $27 \mathrm{kV}$, distance from needle to collector: $15 \mathrm{~cm}$, needle size: 25 gauge, and speed of ejecting solution: $0.5 \mathrm{ml} / \mathrm{h}$ (see Table 1) while the concentration of PLGA solutions was selected as $5 \mathrm{wt} \%, 7 \mathrm{wt} \%$, and $10 \mathrm{wt} \%$, respectively. Results show that the size and shape of the particles depend on PLGA concentration. Specifically, with concentration of $5 \mathrm{wt} \%$ and $7 \mathrm{wt} \%$, the spheres are fabricated (named as PLGA1 MPs and PLGA3 MPs, resp.); however, with concentration of $10 \mathrm{wt} \%$, the fibers were formed (named PLGA3 MPs). Overall the sizes of particles were changed according to changing PLGA concentration. Indeed, when concentration of PLGA increased from $5 \mathrm{wt} \%$ to $7 \mathrm{wt} \%$, the particle sizes increased from approximately $0.2-1 \mu \mathrm{m}$ (Figures 2(a1) and 2(a2)) to $0.2-2 \mu \mathrm{m}$ (Figures 2(b1) and 2(b2)). However, at $10 \mathrm{wt} \%$ of PLGA concentration, the mixing of nanofibers and microparticles formed (Figures 2(c1) and 2(c2)). Therefore, the concentration of $5 \mathrm{wt} \%$ is going to be used for the fabricating core-shell PLGA-CS MPs. 


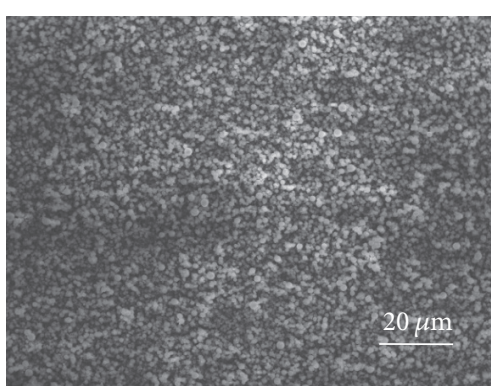

(a1)

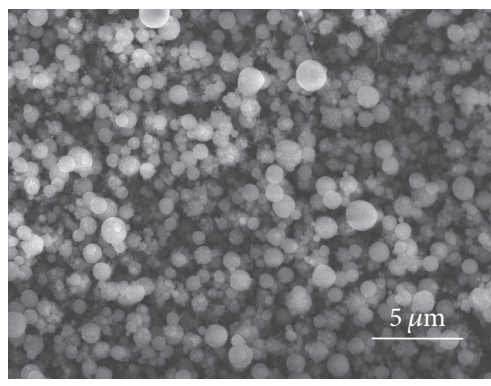

(a2)

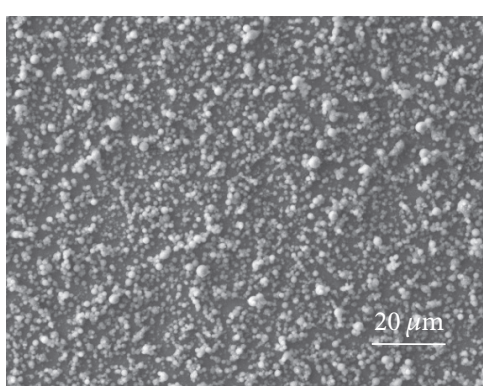

(b1)

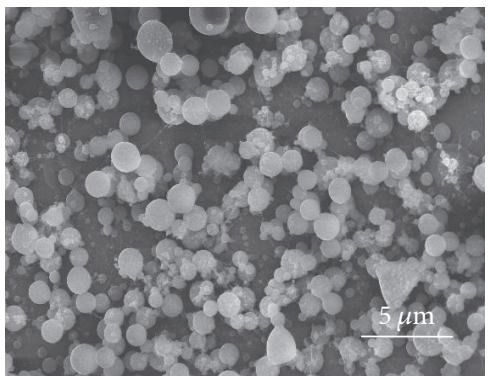

(b2)

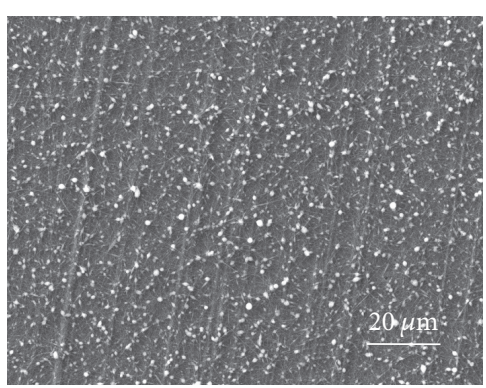

$(\mathrm{c} 1)$

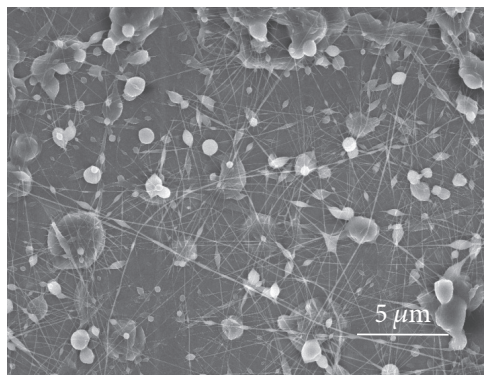

(c2)

FIGURE 2: SEM observation of PLGA MPs in different concentration of PLGA: PLGA1 (a1, a2), PLGA2 (b1, b2), and PLGA3 (c1, c2).

To fabricate the core-shell PLGA-CS MPs, $5 \mathrm{wt} \%$ of PLGA concentration was chosen. The initial electrospinning parameters were fixed as before (distance from needle to collector: $15 \mathrm{~cm}$, needle size: 25 gauge, and speed of ejecting solution: $0.5 \mathrm{ml} / \mathrm{h}$ ). However, the voltage was decreased from $27 \mathrm{kV}$ to $25 \mathrm{kV}$ to optimize the formation of particles. Similar to the investigation of PLGA MPs, here, the PLGA-CS MPs sizes and shapes were controlled solely by the concentration of chitosan. Figure 3 shows SEM morphology of PLGA-CS MPs with $5 \mathrm{wt} \%$ PLGA for different chitosan concentration as follows: $0.01 \mathrm{wt} \%$ (Figures 3(a1) and 3(a2)), $0.15 \mathrm{wt} \%$ (Figures $3(\mathrm{~b} 1)$ and $3(\mathrm{~b} 2)$ ), and $0.3 \mathrm{wt} \%$ (Figures $3(\mathrm{cl})$ and $3(\mathrm{c} 2)$ ), dissolved in $1.5 \mathrm{wt} \%$ acetic acid. For $5 \mathrm{wt} \%$ of PLGA and $0.01 \mathrm{wt} \%$ of chitosan (PLGA-CS1), particle sizes of coreshell PLGA-CS1 MPs were in the range of $0.2-3 \mu \mathrm{m}$. In addition, Figures 3(a1) and 3(a2) show that the chitosan-shells of PLGA-CS1 MPs were melted and deposited around the PLGA-core. However, when the concentration of chitosan increased fifteenfold, that is, for $5 \mathrm{wt} \%$ of PLGA and $0.15 \mathrm{wt} \%$ of chitosan (PLGA-CS2), the particle sizes increased to around 2-10 $\mu \mathrm{m}$, and the surface of PLGA-CS2 MPs became porous. For $5 \mathrm{wt} \%$ of PLGA and $0.3 \mathrm{wt} \%$ of chitosan (PLGACS3), the morphology of PLGA-CS3 MPs was similar to that of PLGA-CS2 MPs with porous structure on the surface and the size of PLGA-CS3 MPs increased dramatically to around 3-20 $\mu \mathrm{m}$.

In addition, the zeta potential of the microparticles increased with higher chitosan concentrations in the external aqueous. It increased from approximately $-4.8 \pm 0.1 \mathrm{mV}$ (chitosan-free PLGA particles) to $+14.9 \pm 0.5 \mathrm{mV}$ when the chitosan concentration in the shell of the core-shell spinneret was $0.01 \mathrm{wt} \%$. The zeta potential increased significantly toward positive charge of $+19.1 \pm 0.4 \mathrm{mV}$ and $+37.0 \pm 0.4 \mathrm{mV}$ when the chitosan concentration was $0.15 \mathrm{wt} \%$ and $0.3 \mathrm{wt} \%$, respectively (Table 2). The higher the degree of zeta potential, the more the stability of particles. This result shows that the increasing of chitosan concentration creates a stability of PLGA-CS MPs in solution [26].

Investigations were conducted to determine if PLGA-CS MPs were indeed composed of PLGA and CS. The presence of PLGA and chitosan in the core-shell PLGA-CS MPs was confirmed by analyzing the presence of specific peaks of single pure materials in the PLGA-CS spectrum such as the strong characteristic absorption bands at about $1746 \mathrm{~cm}^{-1}$ attributed to the stretching vibration of $\mathrm{C}-\mathrm{O}$ bond, and the bands at $1130 \mathrm{~cm}^{-1}$ and $1412 \mathrm{~cm}^{-1}$ arose from $\mathrm{C}-\mathrm{O}$ bond and methyl group C-H bond of PLGA, respectively [27]. Also in Figure 4 the absorptions bands of chitosan are shown: $1094 \mathrm{~cm}^{-1}$ (O-C stretch), $1159 \mathrm{~cm}^{-1}$ (bridge-O stretch), $1313-1481 \mathrm{~cm}^{-1}$ (-CH bend), $1627 \mathrm{~cm}^{-1}$ (C=O stretch), $2923-2867 \mathrm{~cm}^{-1}$ (C-H stretch), $1598-1600 \mathrm{~cm}^{-1}$ (N-H bend), and $3431 \mathrm{~cm}^{-1}$ (O-H stretch) $[28,29]$. Hence, the FT-IR spectrum (Figure 4) confirmed that the presence of chitosan in PLGA-CS MPs and thus PLGA-CS MPs might be fabricated successfully.

To determine if PLGA-CS MPs were indeed coated by chitosan, the fluorescent image of chitosan, optical images of PLGA MPs and PLGA-CS MPs, and weight of chitosan in PLGA-CS MPs were observed and showed in Figure 5. The series fluorescent images (Figures $5(\mathrm{a})-5(\mathrm{~d})$ ) proved that the PLGA MPs were coated by a thin layer of chitosan. Optical images show that the surface of PLGA-CS MP was rough (Figure 5(f)) compared to the smooth surface of PLGA MP (Figure 5(e)). Figure 5(g) is the graph of weight change of chitosan, which shows that chitosan weight increases if the 


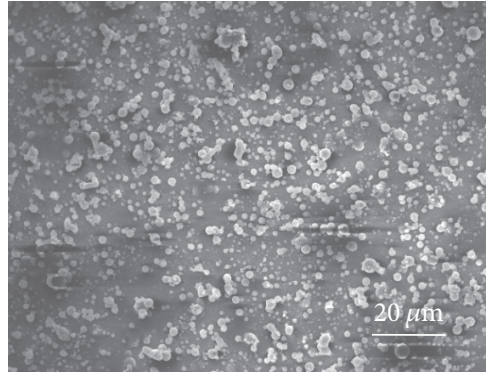

(a1)

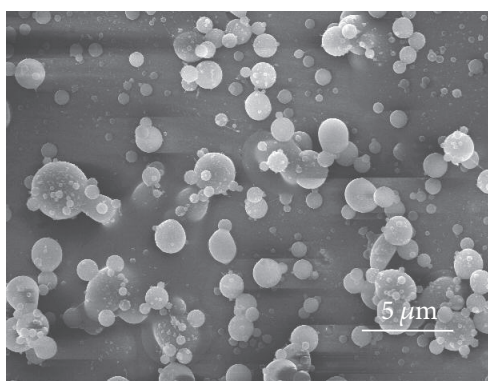

(a2)

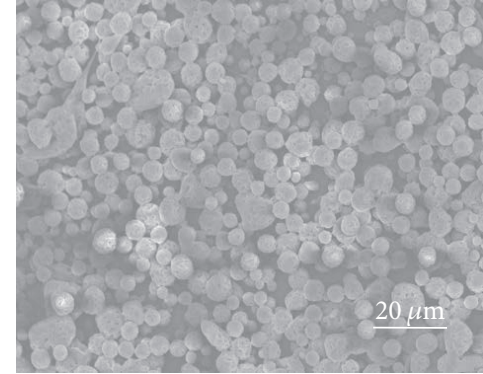

(b1)

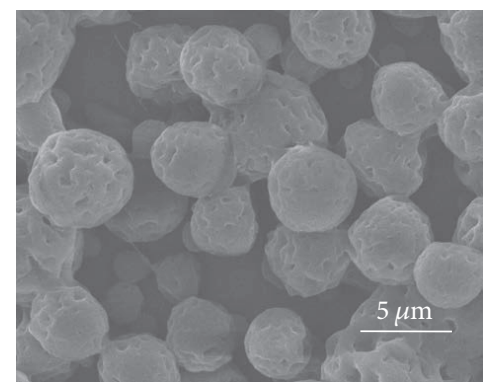

(b2)

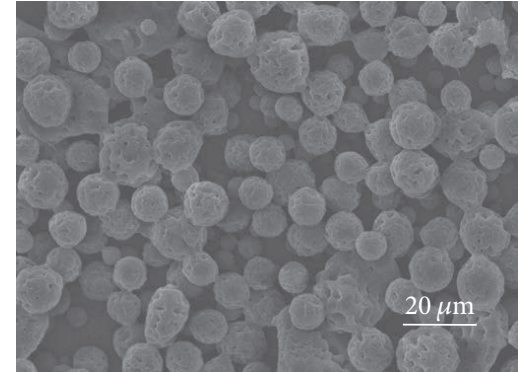

(c1)

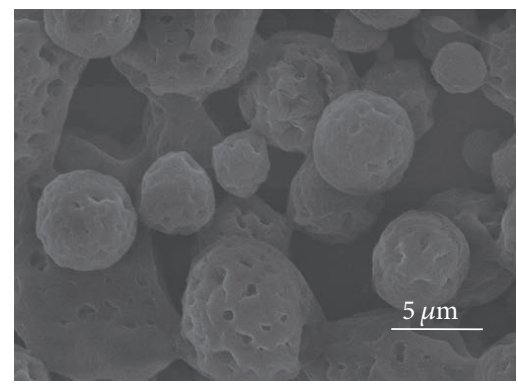

(c2)

FIGURE 3: SEM observation of PLGA-CS MPs in different concentration of chitosan: PLGA-CS1 (a1, a2), PLGA-CS2 (b1, b2), and PLGA-CS3 $(\mathrm{c} 1, \mathrm{c} 2)$.

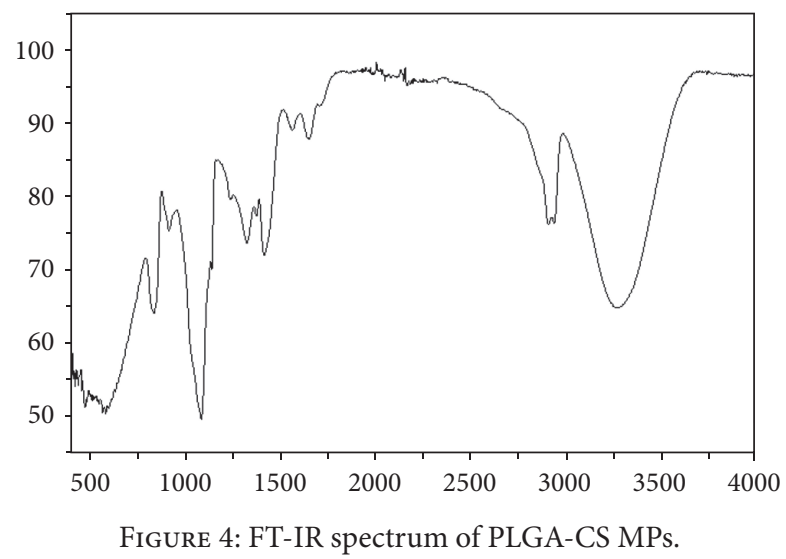

concentration of chitosan increases (PLGA-CS3 > PLGA-CS2 $>$ PLGA-CS1).

The cytotoxicity test is important for new biomaterials to ensure their biocompatibility before conducting in vitro tests. Although both PLGA and CS are known for excellent biocompatibility, in this study, the cytotoxicity of PLGA, PLGA-CS1, PLGA-CS2, and PLGA-CS3 microparticles was examined using MTT assay to ensure safe method. MTT assay is a colorimetric assay that measures the optical density of purple color after the crystallized formazan dyes are dissolved using DMSO. The crystallized formazan was created by yellow MTT solution. The principle of MTT can be briefly described as follows: the MTT enters the cells and passes into the mitochondria where it is reduced to an insoluble and colored (dark purple) formazan product. Since the reduction of MTT can only occur in metabolically active cells, the level of activity is a measure of the viability of the cells. According to the ISO-10993-5, the MTT assay refers to MTT cytotoxicity employed to evaluate new composite materials as previously reported [20]. In this investigation, MTT assay was employed to examine the cytotoxicity and cell proliferation on PLGA and PLGA/CS MPs. The results showed that all of samples had excellent biocompatibility. Indeed, the cell viability of the $100 \%$ extracted solution for all samples (PLGA, PLGA-CS1, PLGA-CS2, and PLGA-CS3) was over $80 \%$ and not significantly different from each other as shown in Figure 6. Thus, the cytotoxicity test revealed that all samples have nontoxic. Washing steps to remove residual solvent are unnecessary in this method. The products are therefore safe for use in biomedical applications.

From the cell proliferation test, Figure 7 shows the optical density of purple color that is produced by DMSO after dissolving the deposited crystallized formazan dyes on samples $[23,24]$. Because MTT detects the activity power of the cells, the results of MTT assay in this examination can be used to evaluate the cell health. Therefore, the viability of cells on the PLGA, PLGA-CS1, PLGA-CS2, and PLGACS3 MPs could be compared using the MTT assay methods [20]. In this examination, microparticles were placed on the aluminum foil and were cultured for 1, 3, 7, and 14 days in static environments in order to observe the growth behavior of the cells as shown in Figure 7. Overall, in comparison with the PLGA MPs group, the optical density of the PLGACS MPs groups decreased when the chitosan concentration increased. Thus results are similar with several reports which indicated polysaccharides such as chitosan derivatives were able to inhibit cell proliferation. However, the investigator can 


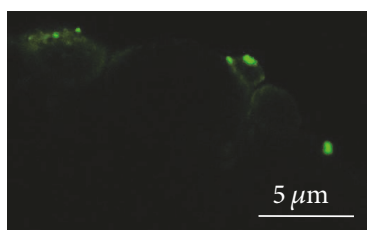

(a)

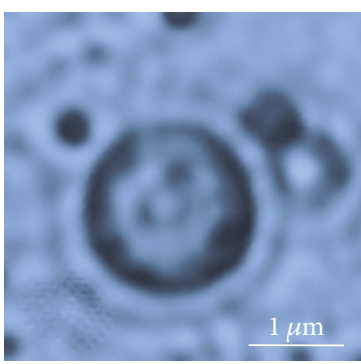

(e)

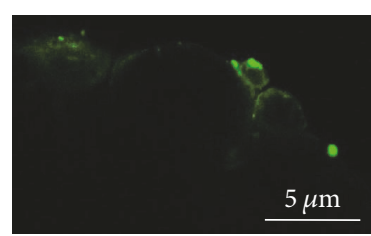

(b)

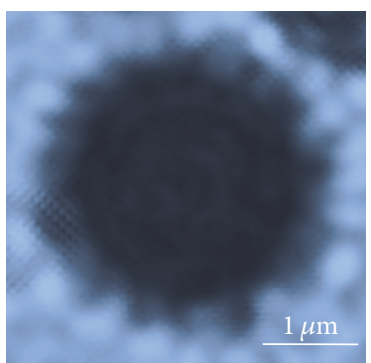

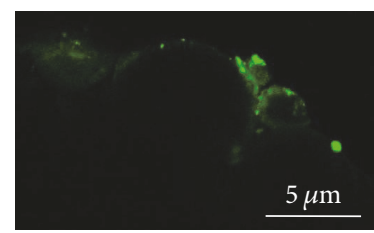

(c)

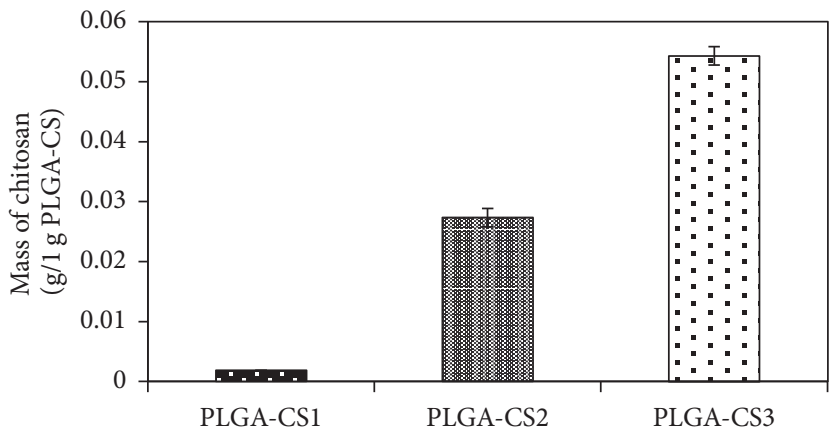

PLGA-CS1

PLGA-CS2

PLGA-CS3

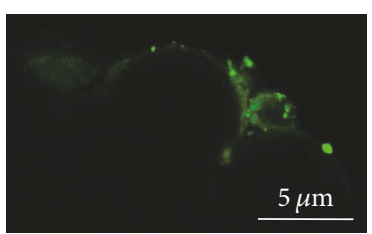

(d)

(f)

(g)

FIGURE 5: Series of images of fluorescein-labeled chitosan coating PLGA was visualized by confocal laser scanning microscopy (a-d). Microscope observation of PLGA MP (e) and PLGA-CS2 MP (f) immersed in distilled water for 24 hours. Mass of chitosan coated PLGA MPs in 1 gram of PLGA-CS MPs.

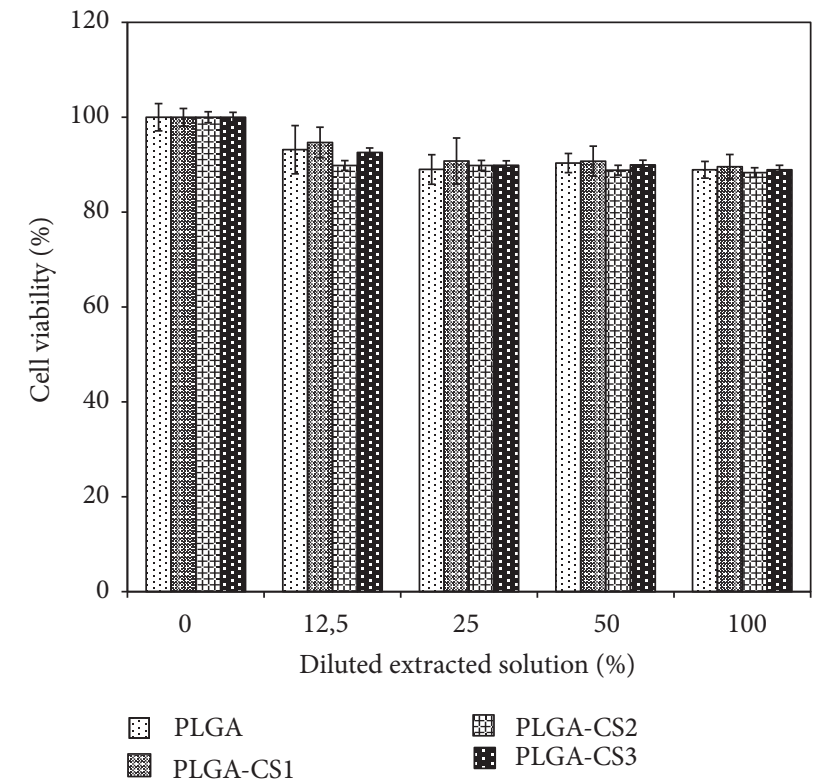

FIgURE 6: Percentage of cell viability on the series of the diluted extracted solution of PLGA MPs and PLGA-CS MPs.

manipulate and take advantage of this for making a stable carrier to carry an anticancer drug [30].

\section{Conclusion}

In this study, the core-shell PLGA-CS MPs have been fabricated successfully using electrospinning machine. The results

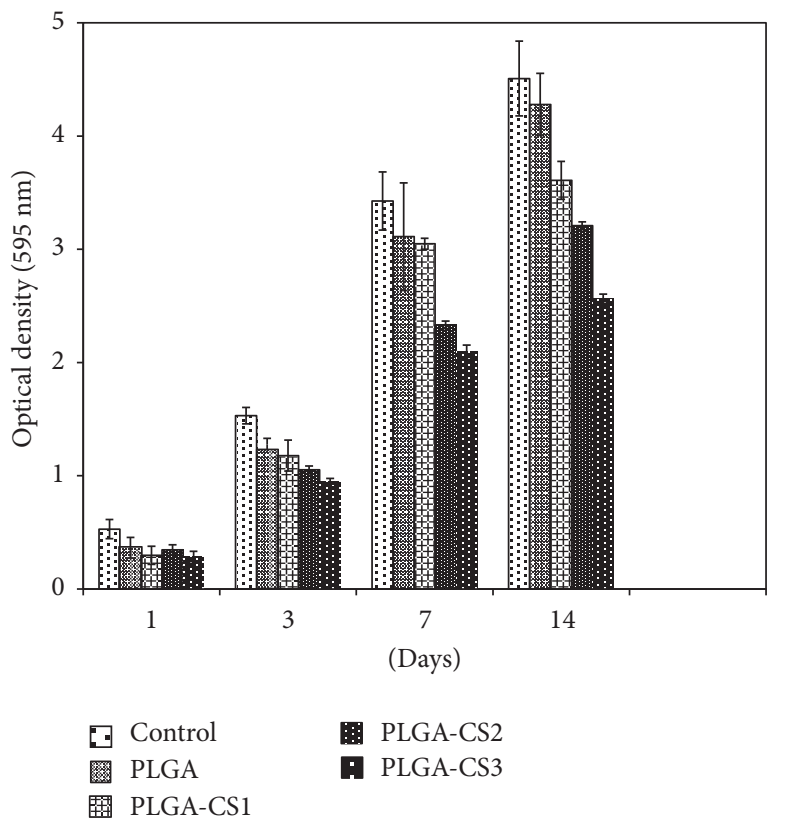

FIGURE 7: Cellular proliferation on the control, PLGA MPs, and PLGA-CS MPs 1, 3, 7, and 14 days cultured.

revealed that the success of PLGA MPs fabrication was determined by concentration of PLGA solution (below $10 \mathrm{wt} \%$ ); the thickness of the chitosan shell can be controlled by the concentration of chitosan. However, PLGA-CS MPs show excellent biocompatibility and cell proliferation. Hence, the 
PLGA MPs or PLGA-CS MPs produced by electrospinning machine promise wide and high potential applications in biomedical fields due to its convenience, cost effectiveness, and excellent biocompatibility.

\section{Conflicts of Interest}

The authors declare that there are no conflicts of interest regarding the publication of this paper.

\section{Acknowledgments}

The research facility was supported by Vietnam National University, Ho Chi Minh City, under Grant no. 1161/QĐĐHQG-KHCN.

\section{References}

[1] T.-Q. Bao, N.-T. Hiep, Y.-H. Kim, H.-M. Yang, and B.-T. Lee, "Fabrication and characterization of porous poly(lactic-coglycolic acid) (PLGA) microspheres for use as a drug delivery system," Journal of Materials Science, vol. 46, no. 8, pp. 25102517, 2011.

[2] C. Vilos and L. A. Velasquez, "Therapeutic strategies based on polymeric microparticles," Journal of Biomedicine and Biotechnology, vol. 2012, Article ID 672760, 2012.

[3] M. Figueiredo and R. Esenaliev, "PLGA Nanoparticles for Ultrasound-Mediated Gene Delivery to Solid Tumors," Journal of Drug Delivery, vol. 2012, pp. 1-20, 2012.

[4] A. Seth and D. S. Katti, "A one-step electrospray-based technique for modulating morphology and surface properties of poly(lactide-co-glycolide) microparticles using Pluronics ${ }^{\circledR,}$," International Journal of Nanomedicine, vol. 7, pp. 5129-5136, 2012.

[5] S. Fischer, C. Foerg, S. Ellenberger, H. P. Merkle, and B. Gander, "One-step preparation of polyelectrolyte-coated PLGA microparticles and their functionalization with model ligands," Journal of Controlled Release, vol. 111, no. 1-2, pp. 135-144, 2006.

[6] N. Nafee, S. Taetz, M. Schneider, U. F. Schaefer, and C.-M. Lehr, "Chitosan-coated PLGA nanoparticles for DNA/RNA delivery: effect of the formulation parameters on complexation and transfection of antisense oligonucleotides," Nanomedicine: Nanotechnology, Biology, and Medicine, vol. 3, no. 3, pp. 173-183, 2007.

[7] L. Chronopoulou, M. Massimi, M. F. Giardi et al., "Chitosancoated PLGA nanoparticles: a sustained drug release strategy for cell cultures," Colloids and Surfaces B: Biointerfaces, vol. 103, pp. 310-317, 2013.

[8] F. Ganji and M. J. Abdekhodaie, "Chitosan-g-PLGA copolymer as a thermosensitive membrane," Carbohydrate Polymers, vol. 80, no. 3, pp. 740-746, 2010.

[9] S.-E. Jin, C.-K. Kim, and Y.-B. Kim, "Cellular delivery of cationic lipid nanoparticle-based SMAD3 antisense oligonucleotides for the inhibition of collagen production in keloid fibroblasts," European Journal of Pharmaceutics and Biopharmaceutics, vol. 82, no. 1, pp. 19-26, 2012.

[10] S. Jafarinejad, K. Gilani, E. Moazeni, M. Ghazi-Khansari, A. R. Najafabadi, and N. Mohajel, "Development of chitosan-based nanoparticles for pulmonary delivery of itraconazole as dry powder formulation," Powder Technology, vol. 222, pp. 65-70, 2012.
[11] Y.-Y. Yang, H.-H. Chia, and T.-S. Chung, "Effect of preparation temperature on the characteristics and release profiles of PLGA microspheres containing protein fabricated by doubleemulsion solvent extraction/evaporation method," Journal of Controlled Release, vol. 69, no. 1, pp. 81-96, 2000.

[12] M. L. Manca, G. Loy, M. Zaru, A. M. Fadda, and S. G. Antimisiaris, "Release of rifampicin from chitosan, PLGA and chitosan-coated PLGA microparticles," Colloids and Surfaces B: Biointerfaces, vol. 67, no. 2, pp. 166-170, 2008.

[13] L.-H. Hung, S.-Y. Teh, J. Jester, and A. P. Lee, "PLGA micro/nanosphere synthesis by droplet microfluidic solvent evaporation and extraction approaches," Lab on a Chip, vol. 10, no. 14, pp. 1820-1825, 2010.

[14] L. Casettari, E. Castagnino, S. Stolnik, A. Lewis, S. M. Howdle, and L. Illum, "Surface characterisation of bioadhesive PLGA/chitosan microparticles produced by supercritical fluid technology," Pharmaceutical Research, vol. 28, no. 7, pp. 16681682, 2011.

[15] R. Jalil and J. R. Nixon, "Biodegradable poly(lactic acid) and poly(lactide-co-glycolide) microcapsules: problems associated with preparative techniques and release properties," Journal of Microencapsulation, vol. 7, no. 3, pp. 297-325, 1990.

[16] B. Almería, W. Deng, T. M. Fahmy, and A. Gomez, "Controlling the morphology of electrospray-generated PLGA microparticles for drug delivery," Journal of Colloid and Interface Science, vol. 343, no. 1, pp. 125-133, 2010.

[17] L. T. Son and K. Takaomi, "Hollow-fiber membrane absorbents embedded molecularly imprinted polymeric spheres for bisphenol A target," Journal of Membrane Science, vol. 384, no. 1-2, pp. 117-125, 2011.

[18] C. Feng, K. C. Khulbe, T. Matsuura, S. Tabe, and A. F. Ismail, "Preparation and characterization of electro-spun nanofiber membranes and their possible applications in water treatment," Separation and Purification Technology, vol. 102, pp. 118-135, 2013.

[19] R. Håkanson, L.-I. Larsson, and F. Sundler, "Fluorescamine: A novel reagent for the histochemical detection of amino groups," Histochemistry, vol. 39, no. 1, pp. 15-23, 1974.

[20] N. T. Hiep and B.-T. Lee, "Electro-spinning of PLGA/PCL blends for tissue engineering and their biocompatibility," Journal of Materials Science: Materials in Medicine, vol. 21, no. 6, pp. 1969-1978, 2010.

[21] T. H. Nguyen and B. T. Lee, "Fabrication and characterization of cross-linked gelatin electro-spun nano-fibers," Journal of Biomedical Science and Engineering, vol. 3, no. 12, pp. 1117-1124, 2010.

[22] T.-H. Nguyen and B.-T. Lee, "The effect of cross-linking on the microstructure, mechanical properties and biocompatibility of electrospun polycaprolactone-gelatin/PLGA-gelatin/PLGAchitosan hybrid composite," Science and Technology of Advanced Materials, vol. 13, no. 3, Article ID 035002, 2012.

[23] T.-H. Nguyen, T. Q. Bao, I. Park, and B.-T. Lee, "A novel fibrous scaffold composed of electrospun porous poly( $\varepsilon$-caprolactone) fibers for bone tissue engineering," Journal of Biomaterials Applications, vol. 28, no. 4, pp. 514-528, 2013.

[24] T.-H. Nguyen and B.-T. Lee, "In vitro and in vivo studies of rhBMP2-coated PS/PCL fibrous scaffolds for bone regeneration," Journal of Biomedical Materials Research-Part A, vol. 101, no. 3, pp. 797-808, 2013.

[25] A. Grenha, C. Remuñán-López, E. L. S. Carvalho, and B. Seijo, "Microspheres containing lipid/chitosan nanoparticles 
complexes for pulmonary delivery of therapeutic proteins," European Journal of Pharmaceutics and Biopharmaceutics, vol. 69, no. 1, pp. 83-93, 2008.

[26] T. A. Ahmed and B. M. Aljaeid, "Preparation, characterization, and potential application of chitosan, chitosan derivatives, and chitosan metal nanoparticles in pharmaceutical drug delivery," Drug Design, Development and Therapy, vol. 10, pp. 483-507, 2016.

[27] M. V. Jose, V. Thomas, D. R. Dean, and E. Nyairo, "Fabrication and characterization of aligned nanofibrous PLGA/Collagen blends as bone tissue scaffolds," Polymer, vol. 50, no. 15, pp. 3778-3785, 2009.

[28] M. Monier, Y. Wei, A. A. Sarhan, and D. M. Ayad, "Synthesis and characterization of photo-crosslinkable hydrogel membranes based on modified chitosan," Polymer, vol. 51, no. 5, pp. 10021009, 2010.

[29] J. Brugnerotto, J. Lizardi, F. M. Goycoolea, W. Argüelles-Monal, J. Desbrières, and M. Rinaudo, "An infrared investigation in relation with chitin and chitosan characterization," Polymer, vol. 42, no. 8, pp. 3569-3580, 2001.

[30] M. Jiang, H. Ouyang, P. Ruan et al., "Chitosan derivatives inhibit cell proliferation and induce apoptosis in breast cancer cells," Anticancer Research, vol. 31, no. 4, pp. 1321-1328, 2011. 

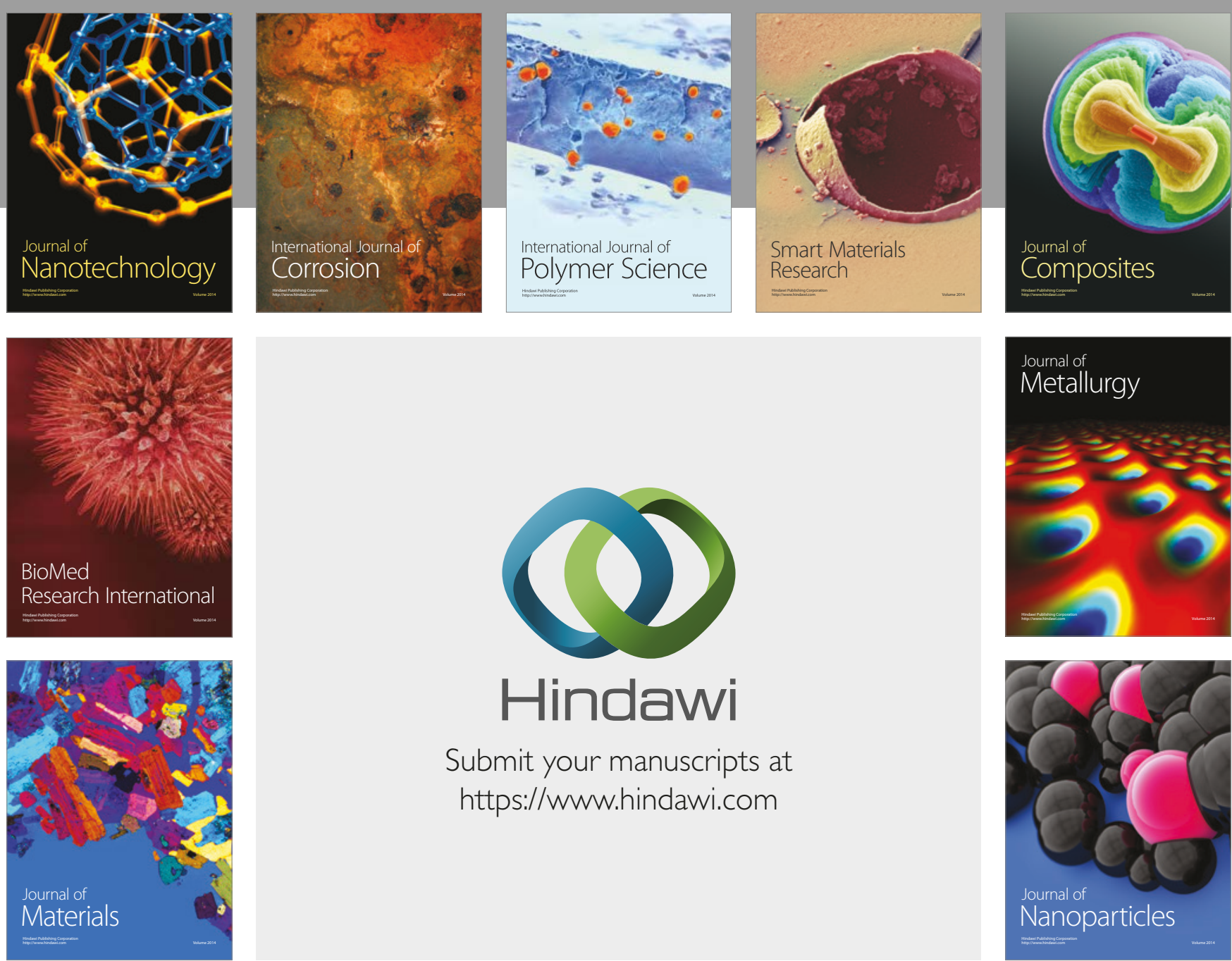

\section{Hindawi}

Submit your manuscripts at

https://www.hindawi.com
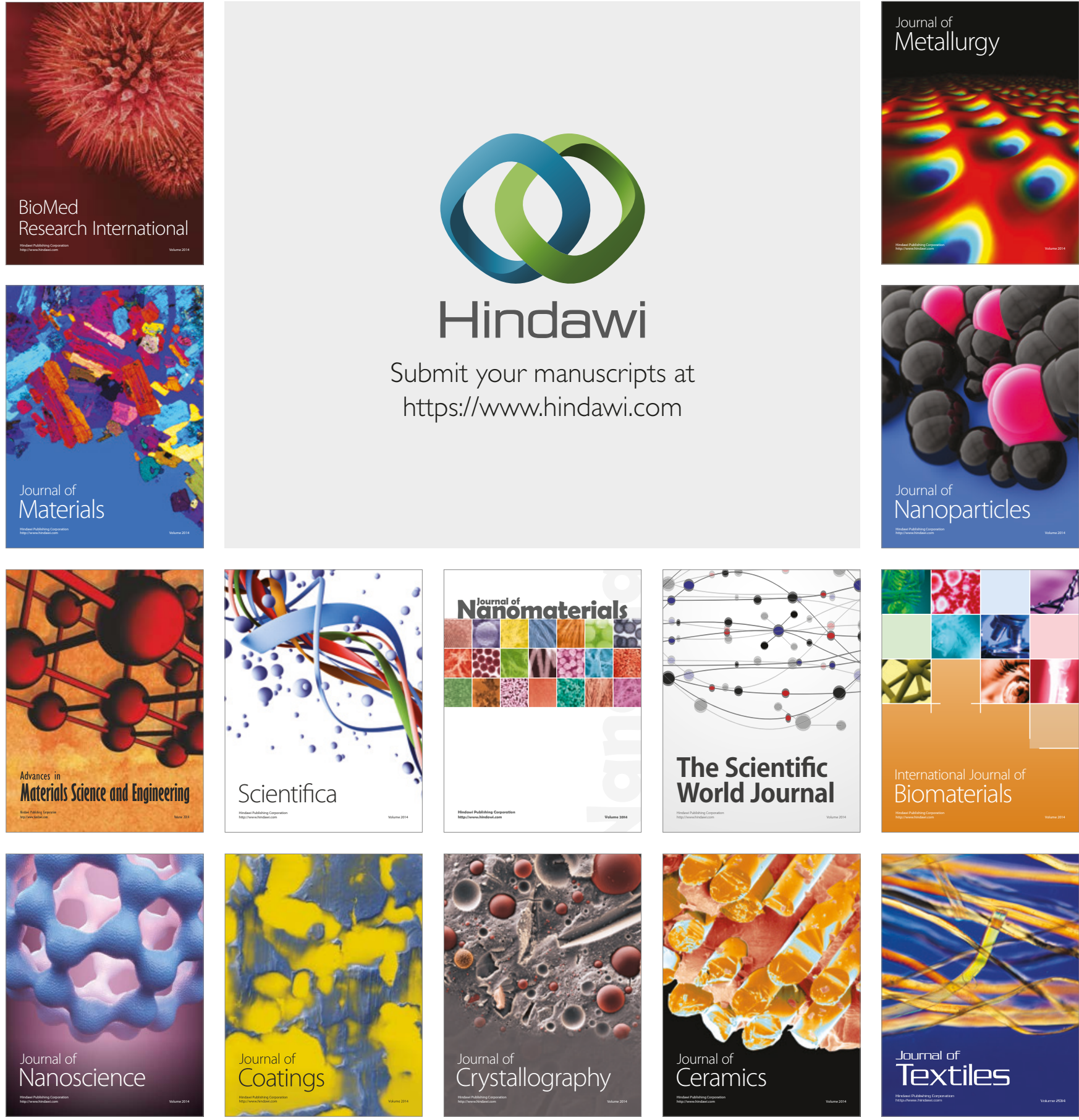

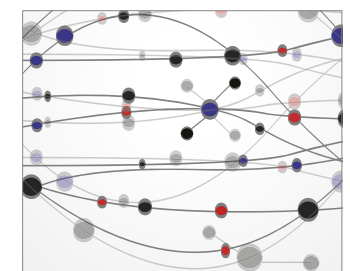

The Scientific World Journal
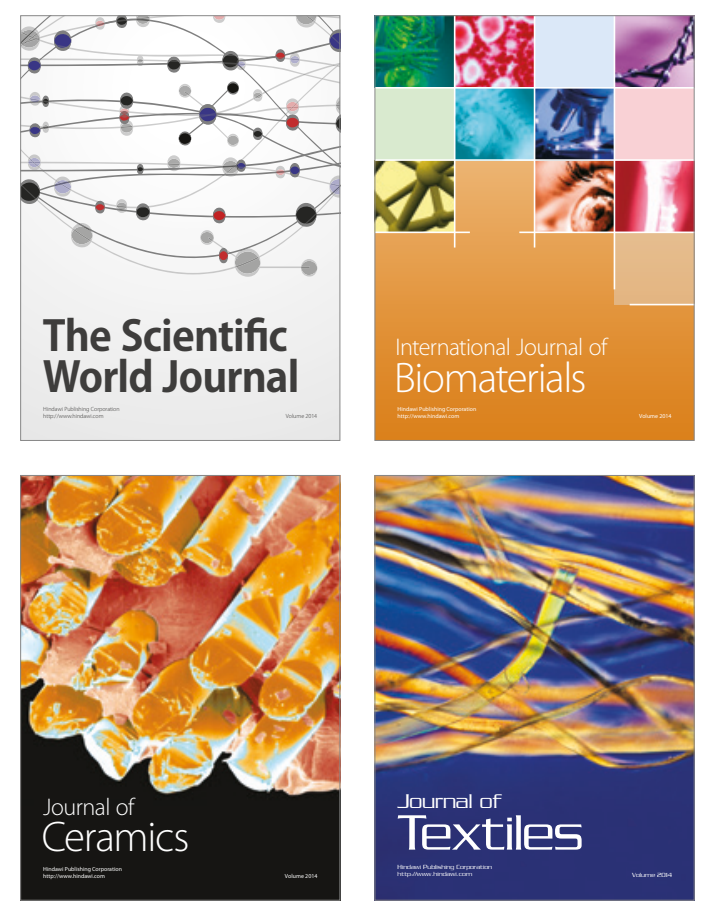